\title{
The Cartwheel galaxy with XMM-Newton
}

\author{
E. Crivellari, A. Wolter, and G. Trinchieri
}

\begin{abstract}
INAF - Osservatorio Astronomico di Brera, via Brera 28, 20121 Milano, Italy
\end{abstract}
e-mail: anna.wolter@brera.inaf.it

Received 25 July 2008 / Accepted 2 April 2009

\begin{abstract}
Aims. The extreme environment provided by the Cartwheel ring is analyzed to study its X-ray and optical-UV properties. We compare the Cartwheel with the other members of its group and study the system as a whole in the X-ray band.

Methods. We analyze the data of the Cartwheel galaxy obtained with XMM-Newton in two different periods (December 2004 and May 2005). We focus on the X-ray properties of the system and use the OM data to obtain additional information in the optical and UV bands. Each dataset is analyzed separately to study source variability and summed together to study fainter and extended sources. Results. We detect a total of 8 sources associated with the Cartwheel galaxy and three in its vicinity, including G1 and G2, all at $L_{\mathrm{X}} \geq 10^{39} \mathrm{erg} \mathrm{s}^{-1}$, that is the Ultra Luminous X-ray (ULX) source range. The brightest ULX source has been already discussed elsewhere. The spectra of the next three brightest ULX are well fitted by a power-law model with a mean photon index of $\sim 2$. We compare the XMM-Newton and Chandra datasets to study the long-term variability of the sources. At least three sources vary in the 5 months between the two XMM-Newton observations and at least four in the 4-year timeframe between Chandra and XMM-Newton observations. One Chandra source disappears and a new one is detected by XMM-Newton in the ring. Optical-UV colors of the Cartwheel ring are consistent with a burst of star formation that is close to reaching its maximum, yielding a mean stellar age of about 40 Myr. The inferred variability and age suggest that high mass X-ray binaries are the counterparts to the ULX sources. The 3 companion galaxies have luminosities in the range $10^{39-40} \mathrm{erg} / \mathrm{s}$ consistent with expectations. The hot gas of the Cartwheel galaxy is luminous and abundant (a few $10^{8} M_{\odot}$ ) and is found both in the outer ring, and in the inner part of the galaxy, behind the shock wave front. We also detect gas in the group with $L_{\mathrm{X}} \sim 10^{40} \mathrm{erg} \mathrm{s}^{-1}$.
\end{abstract}

Key words. galaxies: individual: Cartwheel - X-rays: binaries - X-rays: galaxies - X-rays: ISM

\section{Introduction}

The Cartwheel galaxy is a prototypical example of a ring galaxy, located in a small and compact group of 4 members (SCG 00353357 - Iovino 2002) at redshift $z=0.03$. The group has a velocity dispersion of $\sim 400 \mathrm{~km} \mathrm{~s}^{-1}$ (Taylor \& Atherton 1984) and a radius of $60 \mathrm{kpc}\left(R_{\mathrm{G}}=1.7^{\prime}\right)$. However, three of its members (G1, G2, and Cartwheel itself) are in a tight configuration of $<1^{\prime}$ radius, while $\mathrm{G} 3$, the most likely cause of the impact that led to the formation of the Cartwheel (Higdon 1996), is situated $\sim 3^{\prime}(\sim 100 \mathrm{kpc})$ to the North. The impact with G3 is likely to have transformed a normal spiral into the Cartwheel galaxy today with its prominent elliptical rings, the outer one with major axis $\sim 80^{\prime \prime}(45 \mathrm{kpc})$ and the inner one $\sim 18^{\prime \prime}(10 \mathrm{kpc})$. Optically emitting filaments, called "spokes", connect the two rings.

The Cartwheel galaxy was observed at many wavelengths, from radio (Higdon 1996), infrared (Marcum et al. 1992; Appleton \& Struck-Marcell 1987), optical (Theys \& Spiegel 1976; Fosbury \& Hawarden 1977; Struck et al. 1996), to ultraviolet (Gil de Paz et al. 2007) and X-ray bands. At every wavelength studied the emission from the outer ring was found to be very high, as exemplified in Fig. 1 where X-ray and opticalUV images of the Cartwheel and its surroundings are shown. The first observation in the X-ray band, obtained with ROSAT in 1998, already revealed the prominence of the ring (Wolter et al. 1999). Later, in 2001, Chandra resolved a number of ULXs in the southern part of the ring, due to its excellent angular resolution ( 0.5", Wolter \& Trinchieri 2004; Gao et al. 2003).
We present XMM-Newton data that we use to study the ULXs in the ring, the diffuse emission of the galaxy and the hot-gas group component. In Sect. 2, we present the data; in Sect. 3, we discuss the analysis of both the X-ray and optical/UV data; in Sects. 4-7, we describe our results; and in Sect. 8, we summarize our findings. We assume $H_{0}=75 \mathrm{~km} \mathrm{~s}^{-1} \mathrm{Mpc}^{-1}$ and $z=0.03$, for which $D=122 \mathrm{Mpc}$ and the angular scale is $0.56 \mathrm{kpc} / \mathrm{arcsec}$.

\section{XMM-Newton data}

The Cartwheel galaxy was observed by XMM-Newton on 2004 December 14, 15 (Obs = [101]) for $36 \mathrm{ks}$ and on 2005 May 21, 22 for $60 \mathrm{ks}$ (obs = [201]): in both sets of observations the EPICMOS and EPIC-pn instruments operated in FULL frame mode with the THIN filter applied.

We have presented these X-ray datasets, which we used to study the brightest ULX, source N.10 in Wolter et al. (2006). We summarize the procedure used. To exclude high flaring background periods, we used the XMM-Newton Science Analysis System (SAS - v6.5.0) to extract the light curve of the photons with energy $\geq 10 \mathrm{keV}$, where the signal is mostly due to particles, and we eliminated the periods of time in which the signal was above $0.35 \mathrm{cts} \mathrm{s}^{-1}$ and $1 \mathrm{cts} \mathrm{s}^{-1}$ for EPIC-MOS and EPIC-pn respectively. The final net exposure times were 29/24 ks in the first observation and 50/42 ks in the second one (MOS/pn respectively) corresponding to a $\sim 20-25 \%$ reduction in time. 

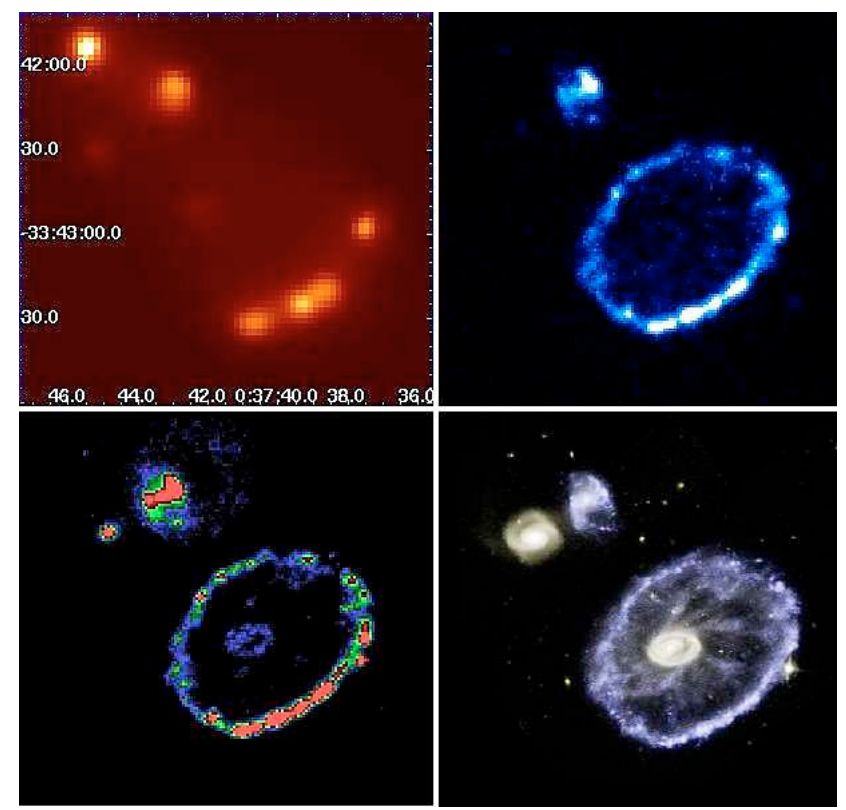

Fig. 1. The Cartwheel group in: X-rays - XMM-Newton - EPIC-MOS (top left). The bright X-ray source in the top left corner is probably a background source; UV - XMM-Newton - OM (UVM2 filter; top right); optical - XMM-Newton - OM ( $U$ filter; bottom left); optical Hubble Space Telescope (bottom right). The Cartwheel and galaxies G1 and G2 are visible, while galaxy G3 is outside the area shown.

The datasets from the two different epochs were both analyzed independently to study the spectra and the variability of point sources, and summed together to improve the statistics for fainter sources and low surface brightness extended emission. For the spectral analysis, we used the EPIC-pn dataset. MOS1 and MOS2 were used only when EPIC-pn data were unavailable.

To analyze the datasets we used, in addition to the $x \mathrm{~mm}$-sas software, XSPEC (v11.3.1), ds9, ciao (v 3.4) ${ }^{1}$.

We also analyzed the optical/UV data obtained with the Optical Monitor onboard XMM-Newton: the first set of observations provides data in the $V, B$, and $U$ filters, while for the second set, UV data are obtained with $U V W 1, U V M 2$, and $U V W 2$ filters. Exposure times, central wavelength of the filter, conversion factors, and zero-point magnitudes are listed in Table $1^{2}$.

\section{Data analysis}

\subsection{EPIC data}

We first studied the point sources of the Cartwheel galaxy. We created 0.3-7 keV images for EPIC-MOS and EPIC-pn separately from both sets of observations, and applied the standard detection algorithm in the area around the Cartwheel simultaneously to all datasets from the same epoch. We detected 6 and 7 sources in the area covered by the disk Cartwheel for the dataset

\footnotetext{
1 http://xmm.esac.esa.int/external/xmm_user_support/ documentation/sas_usg/USG/;

http://heasarc.nasa.gov/docs/xanadu/xspec/manual/ manual.html;

http: //hea-www . harvard. edu/saord/ds9;

http: //cxc.harvard.edu/ciao

2 From

http: //xmm.vilspa.esa.es/external/xmm_user_support/ documentation/uhb/node75.html
}

Table 1. Effective wavelengths and conversion factors (from count rate to flux) of OM filters.

\begin{tabular}{ccccc}
\hline \hline & $\begin{array}{c}\text { Exp. Time } \\
\mathrm{ks}\end{array}$ & $\begin{array}{c}\lambda_{\mathrm{eff}} \\
{[\AA]}\end{array}$ & $\begin{array}{c}\text { Conversion factor } \\
{\left[\mathrm{erg} \mathrm{cm}^{-2} \mathrm{~s}^{-1} \AA^{-1}\right]}\end{array}$ & Zero point \\
\hline$V$ & 8.8 & 5430 & $2.49 \times 10^{-16}$ & 17.963 \\
$B$ & 4.4 & 4500 & $1.29 \times 10^{-16}$ & 19.266 \\
$U$ & 8.0 & 3440 & $1.94 \times 10^{-16}$ & 18.259 \\
$U V W 1$ & 8.8 & 2910 & $4.76 \times 10^{-16}$ & 17.204 \\
$U V M 2$ & 8.8 & 2310 & $2.20 \times 10^{-15}$ & 15.772 \\
$U V W 2$ & 14.4 & 2120 & $5.71 \times 10^{-15}$ & 14.867 \\
\hline
\end{tabular}

of first and second observations, respectively, for a total of 8 sources. We also detected G1 and G2 and another source in the vicinity, which is most likely to be in the background, in both observations. All detected sources are listed in Table 2. To estimate the source strength, we used the final positions given by the detection procedure, but recomputed the net counts in circles of radius $10^{\prime \prime}$ (with the exception of source XMM4, in which $r=8^{\prime \prime}$ to avoid a CCD gap in the EPIC-pn image in obs. [101]), which is smaller than normal but avoids inclusion of nearby sources and a too-large fraction of the surrounding ring-emission. The background for sources XMM9, XMM10, and XMM11, outside the Carthwheel's ring, is computed in a circle of radius $35^{\prime \prime}$ outside the ring and the group area, devoid of sources. For sources in the Cartwheel, we compute the "galaxy" background with two circles of radius $12^{\prime \prime}$ and $10^{\prime \prime}$ including the ring and part of the region inside it but not the detected sources. Since we also intend to compare with Chandra data, the sources are arranged in the order of their Chandra counterparts (from Wolter \& Trinchieri 2004), which are listed in Table 2. Source XMM2=N10 was already presented and discussed in Wolter et al. (2006). It is listed in Table 2 for completeness, but is not discussed further, except when relevant to the other sources. Using a simple power-law spectrum $\left(\Gamma=2.2\right.$ and $n_{\mathrm{H}}=1.9 \times 10^{21} \mathrm{~cm}^{-2}$, which was derived from the sum of detected sources in Chandra, see Wolter \& Trinchieri 2004) and the distance of $122 \mathrm{Mpc}$, we also compute fluxes and the intrinsic luminosity of each source, which we list in Table 2. We confirm the high luminosity of each XMM-Newton detections $\left(\geq 10^{39} \mathrm{erg} / \mathrm{s}\right)$, as already noted from the Chandra results, that places each of them in the range of ULX sources.

We performed spectral analysis for the brightest sources (with about $\geq 100$ net counts). We binned the data so that each final bin has a significance of at least $2 \sigma$ in the net data, and we fitted the spectra with a power-law model including low energy absorption caused by an intervening column with free $N_{\mathrm{H}}$.

We also studied the unresolved component in the galaxy to determine its spectral characteristics and luminosity. We first made a projection of the net counts in the region shown in Fig. 2. We detected a positive signal inside the Cartwheel ring and in the region between the Cartwheel and its two nearby companion galaxies, which appears to represent a contribution from both the body of the Cartwheel and from the group.

To study the spectral characteristics of the unresolved emission in the Cartwheel, we considered both a region corresponding to the ring ("ring"), and one inside it ("in"), as shown in Fig. 3. All detected sources were masked out. We then summed the resulting spectra from the two observations and combined the response matrixes (weighted by the relative exposure times). With the same procedure, we also obtained the spectral data for the companion galaxies, G1 and G2 (G3 has an insufficient 
Table 2. Names, positions, and counts. Background includes region of the Cartwheel ring and inside, but not the detected sources.

\begin{tabular}{|c|c|c|c|c|c|c|c|}
\hline \multirow[t]{2}{*}{ XMM name } & $\begin{array}{ll}\text { RA } & \text { Dec }\end{array}$ & \multicolumn{2}{|c|}{ pn Net counts $(0.3-7 \mathrm{keV})$} & \multirow[t]{2}{*}{ Chandra name } & \multicolumn{2}{|c|}{$\log L_{X} \operatorname{erg} / \mathrm{s}(0.5-2 \mathrm{keV})$} & \multirow[t]{2}{*}{ Detected } \\
\hline & $(\mathrm{J} 2000)$ & {$[101]$} & [201] & & {$[101]$} & {$[201]$} & \\
\hline XMM1 & $00: 37: 40.8-33: 43: 32.5$ & $110.94 \pm 12.94$ & $82.36 \pm 13.14$ & $N 7+9$ & 40.06 & 39.69 & 1:yes; 2:yes \\
\hline XMM2 & $00: 37: 39.4-33: 43: 23.2$ & $209.09 \pm 16.35$ & $184.43 \pm 16.63$ & $N 10$ & 40.33 & 40.04 & 1:yes; $2:$ no \\
\hline XMM3 & $00: 37: 39.0-33: 42: 49.3$ & $12.80 \pm 8.24$ & $16.77 \pm 10.22$ & N11 & 39.12 & 39.00 & 1:yes; 2:yes \\
\hline XMM4* & $00: 37: 39.2-33: 42: 32.8$ & $4.68 \pm 6.18$ & $19.78 \pm 8.45$ & N12 & 38.68 & 39.07 & 1:yes; 2 :yes \\
\hline XMM5 & $00: 37: 39.0-33: 43: 19.2$ & $153.33 \pm 13.89$ & $202.89 \pm 17.15$ & $N 13+14$ & 40.20 & 40.08 & 1:no; 2:yes \\
\hline XMM6 & $00: 37: 37.5-33: 42: 56.9$ & $47.94 \pm 10.23$ & $96.36 \pm 13.66$ & $N 16+17$ & 39.69 & 39.76 & 1:yes; 2:yes \\
\hline XMM7 & $00: 37: 40.9-33: 42: 33.5$ & $<7.40$ & $31.89 \pm 11.09$ & N21 & $<38.38$ & 39.28 & 1: no; 2 :yes \\
\hline XMM8 & $00: 37: 42.2-33: 42: 51.5$ & $13.94 \pm 8.40$ & $30.89 \pm 11.05$ & none & 39.16 & 39.26 & 1:yes; 2:yes \\
\hline XMM9 & $00: 37: 43.1 \quad-33: 42: 07.9$ & $132.42 \pm 12.21$ & $199.00 \pm 15.09$ & $G 1$ & 40.13 & 40.07 & 1:yes; 2:yes \\
\hline XMM10 & $00: 37: 45.1 \quad-33: 42: 28.4$ & $33.53 \pm 7.07$ & $80.41 \pm 10.48$ & G2 & 39.54 & 39.68 & 1:yes; 2 :yes \\
\hline XMM11 & $00: 37: 45.5-33: 41: 52.8$ & $89.53 \pm 10.30$ & $344.41 \pm 19.33$ & СХОЈ003745.6-334151 & 39.96 & 40.55 & 1:yes; 2 :yes \\
\hline
\end{tabular}

${ }^{*}$ Radius $=8 "$ close to pn gap in [101].
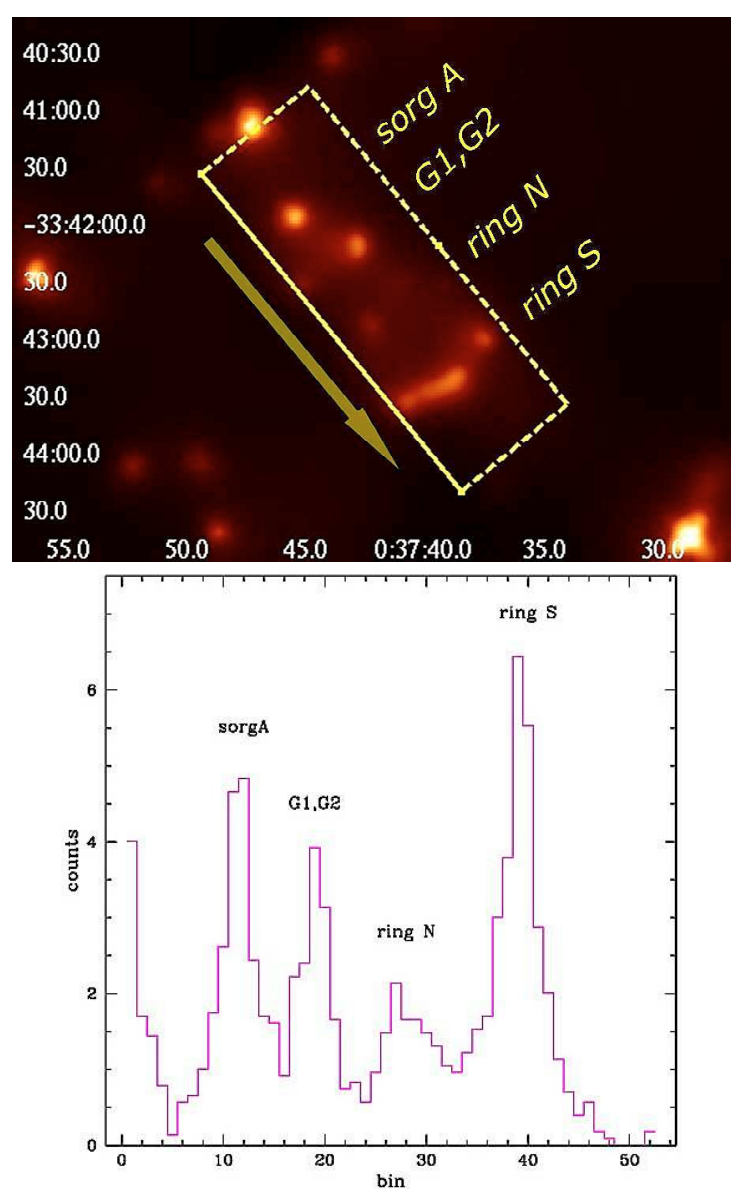

Fig. 2. Left: projection region (thickness $=72^{\prime \prime}$, length $\left.=215^{\prime \prime}\right)$; the background is derived from a box, parallel to this one, displaced to the SE. Right: projection of the background-subtracted counts (in NE-SW direction).

number of counts), and for a region between them, but outside the Cartwheel's ring, where we detected emission from the group.

We used a power-law model to represent an unresolved binary component, while the hot plasma component was described by a mekal model with abundances fixed at $0.5 \times$ solar, since the optical metallicity is low (Fosbury \& Hawarden 1977). The

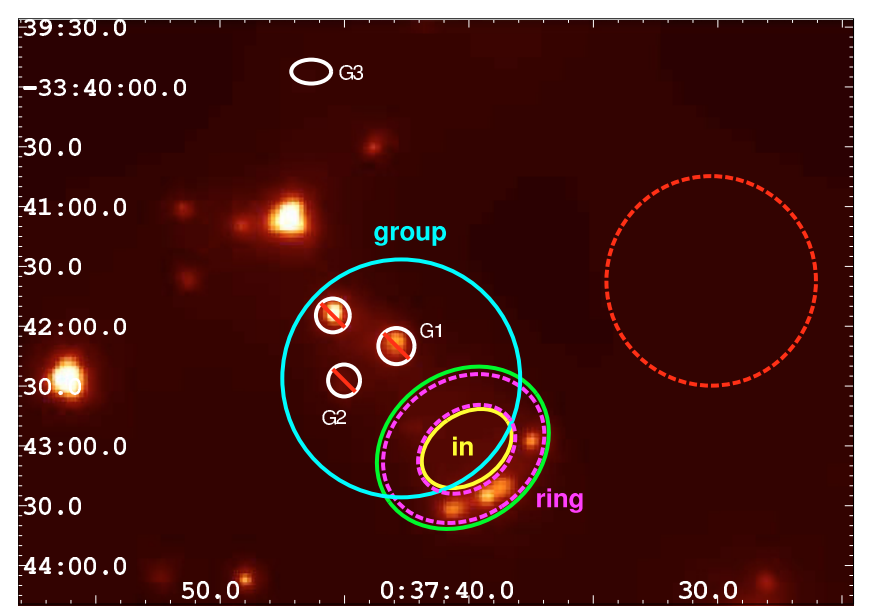

Fig. 3. The $0.3-2 \mathrm{keV}$ MOS1+MOS2 image, with the regions used to extract the spectra and the background region overplotted as labeled. The background region is the dashed circle. All detected sources are excluded in the calculation, but not all the corresponding excluded regions are shown, for clarity.

choice of abundances however had no influence on the final results for $k T$.

The spectral results are discussed in Sects. 4-6 for the point sources, the companion galaxy, and the diffuse emission, respectively.

\subsection{OM data}

OM data were processed in XMM-SAS using the standard parameters described in the XMM-Newton Science Analysis System Users' Guide.

For the brightest sources, we calculated the net counts in a region of radius $2^{\prime \prime}$ for the optical filters, and $3^{\prime \prime}$ for the UV ones, centered on the pixel with the highest signal consistent with the Chandra position. A mean background was evaluated by computing the average surface brightness within different circular regions outside but still close to the galaxy. Given the presence of ghost images, which were more prominent in some filters, we chose different background regions appropriately for each source to minimize the contamination from the ghost images. With the same procedure we also computed net counts from the region 

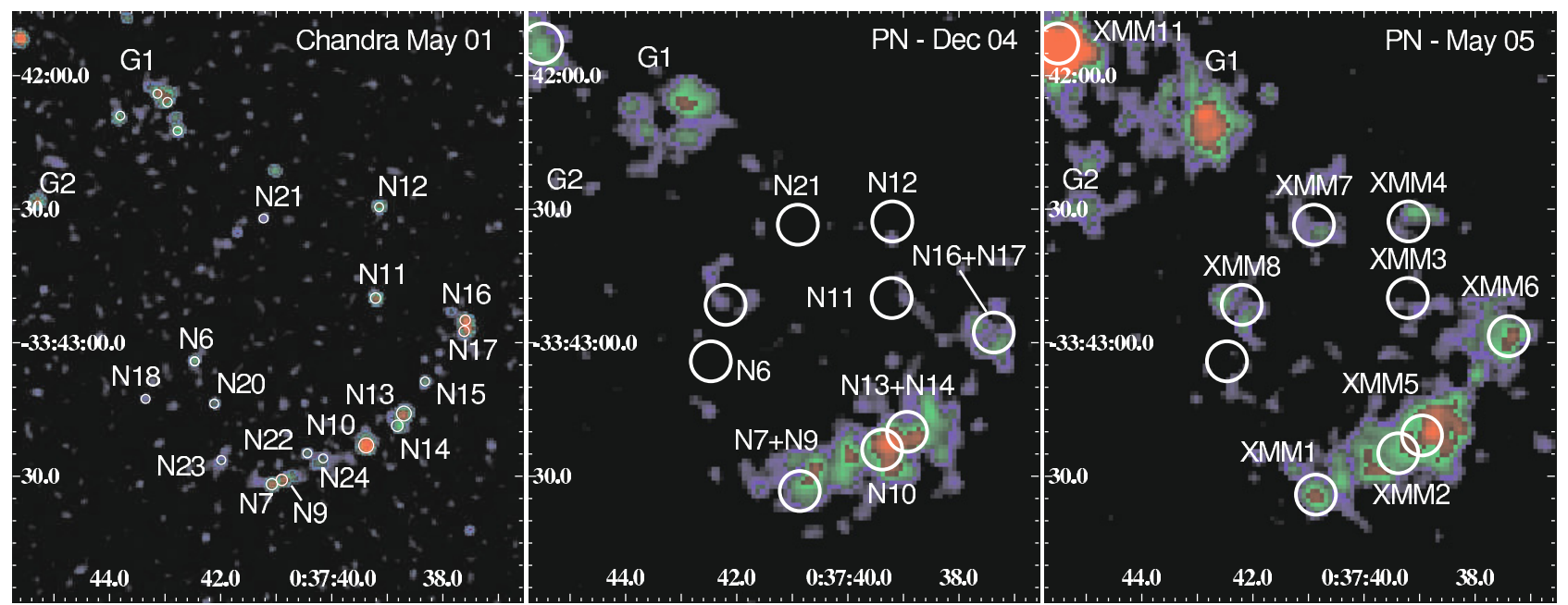

Fig. 4. The three panels represent a zoomed image of the ring on the same scale. Left: $0.3-2 \mathrm{keV}$ Chandra image. Middle and right: $0.3-2 \mathrm{keV}$ EPIC-pn images, smoothed with a 3" Gaussian, of the two epochs respectively. Source names of the detected sources are indicated using Chandra names in the middle panel and using XMM-Newton names in the right panel.

corresponding to the brightest Mid-IR hot spot (an HII region, see Charmandaris et al. 1999) and from the galaxies G1 and G2.

We then used the conversion factors listed in Table 1 to derive fluxes and Vega magnitudes of these sources. Our results are described in Sect. 7.

\section{Point sources and variability}

Seven sources were detected in the Cartwheel ring in the XMMNewton data. One additional source was found in the region inside the ring $(\mathrm{XMM} 3=\mathrm{N} 11)$ and 1 outside $(\mathrm{XMM} 11)$. G1 and G2 were also clearly detected.

A zoomed image of the southern portion of the ring is shown in Fig. 4 and compared with the previous Chandra image of the same region. Because of the lower XMM-Newton resolution $\left(F W H M \sim 6^{\prime \prime}\right)$, many XMM-Newton sources correspond to two Chandra ones.

Five sources (XMM1, XMM3, XMM4, XMM6, XMM8) plus the three sources clearly not associated with the Cartwheel (XMM9, XMM10, and XMM11) are detected in both sets of observations. Source XMM2 is found only in the first dataset, while sources XMM5 and XMM7 were detected in the second set. All sources except XMM8 were previously detected by Chandra, as clearly indicated by the associations listed in Table 2.

We compare XMM-Newton fluxes derived in Sect. 3.1 with Chandra fluxes from 2001. The resulting light curves are plotted in Fig. 5. Given the different spatial resolutions, we summed the contribution from two Chandra sources when more than one was included in the larger XMM-Newton PSF (as indicated by their names). We also estimated the values relative to source N.6, the brightest source detected in the Chandra image and formally undetected in the XMM-Newton datasets. For source XMM8 previously undetected, we assumed a flux at the Chandra epoch equivalent to the lowest detected source in the ring or vicinity as a reasonable upper limit to its flux.

We observed fluctuations in the flux of the sources in the two observations, even if they were not of large amplitude. Sources N.7+9 and N.13+14 show a decrease in count rate, while in N.21 there is an increase. Other sources show fluctuations either way, but they should be considered constant within the uncertainties.

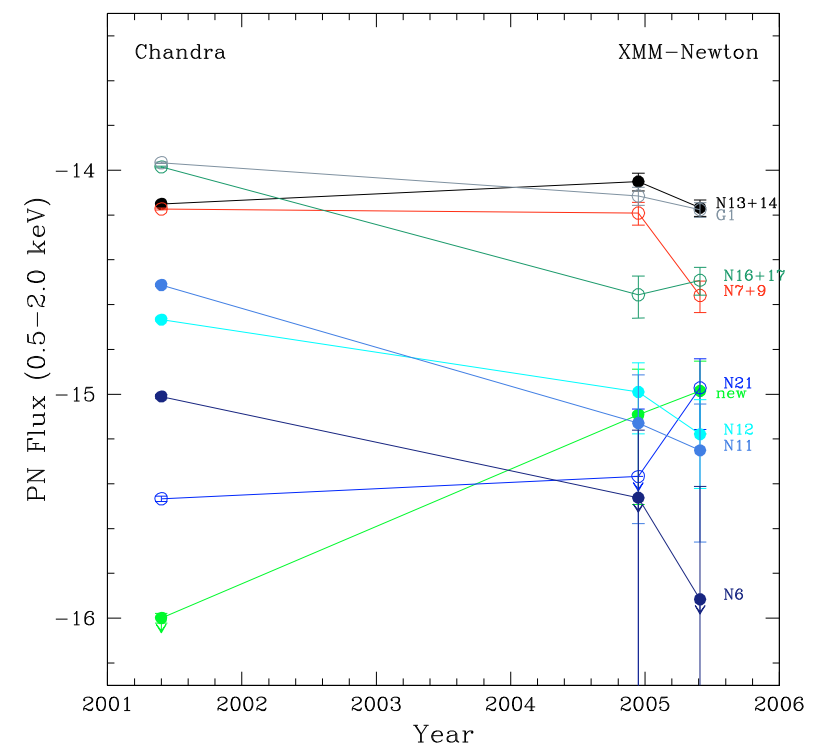

Fig. 5. Comparison of fluxes in the 4-year time-frame covered by Chandra and XMM-Newton. Plotted errorbars include only statistical errors. The same spectral shape is used for all observations, see text for details. Chandra values are from Wolter \& Trinchieri (2004).

We detected evidence of variability on timescales of a few years in several cases: N.16+17, N.11, N.12, N.21, and N.6 varied significantly in brightness between the Chandra and the XMM-Newton first observation, and XMM8 has significantly increased in brightness since 2001.

For the three brightest sources detected with >100 EPICpn counts, we attempted a spectral fit. For consistency with Chandra results, we used a background level measured outside the Cartwheel disk, and therefore we needed to use a complex model to account for different components, since the ring is likely to contribute significantly. We used the model derived from the Chandra data to parameterize the ring emission (mekal with $k T=0.2 \mathrm{keV}$, to account for a hot gas component, and powerlaw with $\Gamma=2.3$ for the unresolved binary population, with the relative normalizations fixed to the Chandra values, Wolter \& Trinchieri 2004), and rescaled it to the area subtended 
Table 3. Spectra, fluxes, and luminosities of the three brightest ULX in the ring.

\begin{tabular}{c|c|cc|cc|cc|cc}
\hline \hline & $\Gamma$ & \multicolumn{2}{|c|}{$n_{\mathrm{H}}$} & \multicolumn{2}{c|}{$\chi^{2}$} & \multicolumn{2}{c|}{ Flux } & \multicolumn{2}{c}{ Lum } \\
& & \multicolumn{2}{|c|}{$\left[\mathrm{cm}^{-2}\right] \times 10^{21}$} & & & $(2-10 \mathrm{keV})\left[\mathrm{erg} \mathrm{cm}^{-2} \mathrm{~s}^{-1}\right] \times 10^{-14}$ & $(2-10 \mathrm{keV})\left[\mathrm{erg} \mathrm{s}^{-1}\right] \times 10^{40}$ \\
& & {$[101]$} & {$[201]$} & {$[101]$} & {$[201]$} & {$[101]$} & {$[201]$} & {$[101]$} & {$[201]$} \\
\hline $\boldsymbol{N 7 + N 9}$ & $\Gamma=1.9$ & $1.7 \pm 0.6$ & $1.8 \pm 0.5$ & 0.35 & 0.34 & $1.59[1.22-1.94]$ & $0.89[0.60-1.20]$ & $2.94[2.32-3.52]$ & $1.62[1.14-1.97]$ \\
$\boldsymbol{N 1 3 + N 1 4}$ & $\Gamma=2.1 / 1.4$ & $2.3 \pm 0.7$ & $0.6 \pm 0.3$ & 0.97 & 0.92 & $1.55[1.21-2.02]$ & $1.51[1.09-1.80]$ & $2.81[2.34-3.76]$ & $2.68[2.11-3.34]$ \\
$\mathbf{N 1 6 + N 1 7}$ & $\Gamma=2.2$ & $2.0 \pm 0.6$ & $2.0 \pm 0.6$ & 0.78 & 1.2 & $0.56[0.34-0.85]$ & $0.71[0.49-0.90]$ & $1.04[0.67-1.38]$ & $1.30[0.89-1.65]$ \\
\hline
\end{tabular}

Table 4. Count rates and luminosities of Cartwheel companion galaxies.

\begin{tabular}{|c|c|c|c|c|c|c|c|}
\hline & \multirow[b]{2}{*}{$\begin{array}{c}\text { EPIC-pn Count rate } \\
(0.3-7 \mathrm{keV})\left[\mathrm{s}^{-1}\right]\end{array}$} & \multirow{2}{*}{$\begin{array}{c}\text { "Gas" } \\
L_{\mathrm{x}}(0.5-2 \mathrm{keV}) / k T \\
{\left[\operatorname{erg~s}^{-1}\right] / \mathrm{keV}}\end{array}$} & \multicolumn{2}{|c|}{ "Stellar" component } & \multirow[b]{2}{*}{$\Gamma$} & \multirow[b]{2}{*}{$\begin{array}{c}n_{\mathrm{H}} \\
{\left[\mathrm{cm}^{-2}\right] \times 10^{20}}\end{array}$} & \multirow[t]{2}{*}{$\chi^{2}$ (d.o.f.) } \\
\hline & & & $\begin{array}{c}L_{\mathrm{x}}(0.5-2 \mathrm{keV}) \\
{\left[\mathrm{erg} \mathrm{s}^{-1}\right]}\end{array}$ & $\begin{array}{c}L_{\mathrm{x}}(2-10 \mathrm{keV}) \\
{\left[\mathrm{erg} \mathrm{s}^{-1}\right]}\end{array}$ & & & \\
\hline G1 & $(6.4 \pm 0.3) \times 10^{-3}$ & $\begin{array}{c}8.6 \pm 0.9) \times 10^{39} / \\
0.22[0.16 \quad 0.28]\end{array}$ & $(2.1 \pm 0.3) \times 10^{40}$ & $(1.9 \pm 0.2) \times 10^{40}$ & $1.86\left[\begin{array}{ll}1.26 & 2.49\end{array}\right]$ & 17.[13. 82] & $0.94(24)$ \\
\hline G1 & idem & - & $(2.4 \pm 0.2) \times 10^{40}$ & $(1.5 \pm 0.2) \times 10^{40}$ & $2.15\left[\begin{array}{ll}1.8 & 2.5\end{array}\right]$ & 9.[3. 16.] & $1.09(26)$ \\
\hline$G 2$ & $(2.9 \pm 0.2) \times 10^{-3}$ & - & $(8.1 \pm 2.1) \times 10^{39}$ & $(5.4 \pm 1.3) \times 10^{39}$ & $2.35\left[\begin{array}{ll}2.06 & 2.98\end{array}\right]$ & $8.9\left[\begin{array}{ll}3.8 & 17.8\end{array}\right]$ & $1.12(16)$ \\
\hline G3 & $*(2.6 \pm 0.9) \times 10^{-4}$ & - & $(3.0 \pm 1.0) \times 10^{39}$ & $(1.9 \pm 0.7) \times 10^{39}$ & 2.15 & 9.0 & \\
\hline
\end{tabular}

Results for G1 are given for both 2 model components (1st line) and single power law (2nd line). ${ }^{*}$ Count rate for G3 is from MOS1+MOS2.

by the source region. The source spectrum was parameterized by a power-law model with low energy absorption. In Table 3, we list the photon index, and the fitted absorption, which is always much greater than Galactic, fluxes and luminosities for the ULX component only, i.e. cleaned of the contribution from the ring parametrized as discussed above.

Source N13+14 deserves closer scrutiny. For example it is possible that the presence of source N.10 at $\sim 10^{\prime \prime}$ from N13+14 prevents the detection of this latter source in the first observation, since it contributes only a small fraction of the emission and is therefore difficult to separate from the much brighter source N.10. In contrast, in obs. [201], N.10 has dimmed (Wolter et al. 2006) and was not formally detected, while N13+14 was. We also note that in the first XMM-Newton observation the source appears brighter than in the Chandra data, which again could be due to contamination from the wings of the brighter source. The spectral data of the two XMM-Newton observations indicate a mild count rate variability and different best-fit values, as shown in Fig. 6: the photon index varies from 2.1 [1.662.66 ] to 1.4 [1.00-1.97], and the absorption coefficient also decreases in the second observation (from $2.3[1.4-3.4] \times 10^{21}$ to $\left.6.5[2.9-11.4] \times 10^{20} \mathrm{~cm}^{-2}\right)^{3}$. Unfortunately, the statistical significance of the two observations is low. However, it appears that the spectral variation is in a sense opposite to that expected from strong contamination from source N.10: the spectral shape of this latter is $\Gamma=1.6$ and should act in an opposite sense to the spectral change observed.

All point sources exhibit some type of variability either on 5-month or 4-year timescales. A few sources vary by up to a factor of $\geq 10$ in flux. This is a clear indication that the $\mathrm{X}$-ray source is an individual accreting object. The statistics of the individual detections do not allow a more precise comparison with e.g. the Galactic black hole spectral-luminosity plane. Variability on timescales of months to years is also observed in other galaxies. In the most studied of them, the Antennae (Zezas et al. 2006), most ULXs exhibit long-term variability, of factors $\sim 2-6$. Source 39 exhibits a strong decline to very faint

\footnotetext{
${ }^{3}$ Note that the flux reported in Fig. 5 is derived by assuming the same spectral shape with $\Gamma=2.2$.
}

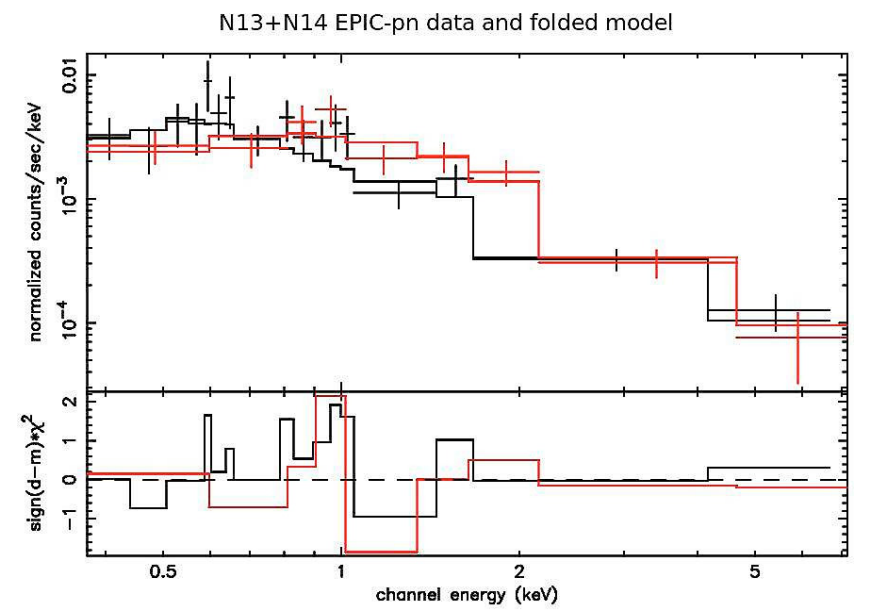

Fig. 6. Spectra of source N13+14; in red: data from 1st observation Dec. 2004 fitted with $\Gamma=2.1$ and $n_{\mathrm{H}}=2.3 \times 10^{21} \mathrm{~cm}^{-2}$; in black: data from 2nd observation - May 2005 fitted with $\Gamma=1.4$ and $n_{\mathrm{H}}=$ $6.5 \times 10^{20} \mathrm{~cm}^{-2}$.

fluxes $\left(\Delta f_{\mathrm{X}}>10\right)$, which could be similar to the behavior of our sources XMM8 and N6.

\section{Companion galaxies}

We extracted the spectra of the companion galaxies from the two EPIC-pn datasets and we coadded the data since we did not expect significant time variability (see also Fig. 5). We attempted to represent the spectrum with a powerlaw model plus a mekal model, to account for both the stellar component and the gas contribution, when present. In Table 4, we summarize the count rates and luminosities of these galaxies.

The spectrum of G1 is well fitted by a single component, an absorbed powerlaw, with slope $\Gamma=2.15$ and $n_{\mathrm{H}}=1.4 \times$ $10^{21} \mathrm{~cm}^{-2}$, which implies a total $L_{\mathrm{X}}(0.5-10)=3.9 \times 10^{40} \mathrm{erg} \mathrm{s}^{-1}$. Similar parameters characterize the G2 spectrum: $\Gamma=2.4$ and $n_{\mathrm{H}}=9 . \times 10^{20} \mathrm{~cm}^{-2}$, for a total $L_{\mathrm{X}}(0.5-10)=1.4 \times 10^{40} \mathrm{erg} \mathrm{s}^{-1}$, 


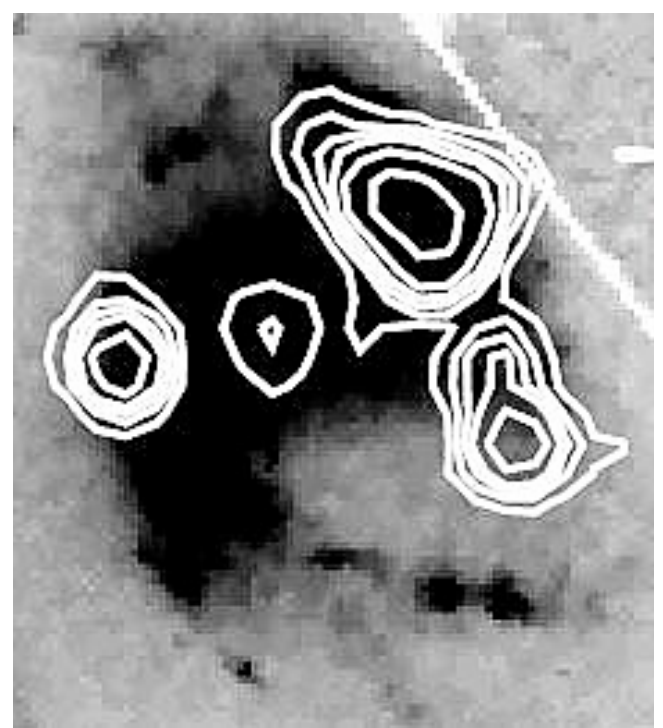

Fig. 7. The X-ray contours in the $0.3-7 \mathrm{keV}$ Chandra image overplotted on the optical HST image (adapted from Wolter \& Trinchieri 2004).

with larger uncertainties because of the smaller number statistics. In both cases, as for the Cartwheel galaxy itself, the lineof-sight Galactic value of $n_{\mathrm{H}}=1.94 \times 10^{20} \mathrm{~cm}^{-2}$ (Dickey \& Lockman 1990) is about a factor of 10 lower, which is indicative of absorption within the galaxies.

While formally not required by the fitting procedure, the addition of a mekal component to the spectral fit of the G1 data improves the distribution of the residuals, which show an excess at $0.6-0.8 \mathrm{keV}$ above the single power law model. This component has a temperature of $k T=0.22 \mathrm{keV}$ and an unabsorbed luminosity of $L_{\mathrm{X}}^{0.5-2}=8.6 \times 10^{39} \mathrm{erg} / \mathrm{s}$ (see Table 4 ).

The position of G3 coincides with a bad column in EPIC-pn in both observations. Therefore we used the MOS1 plus MOS2 data (a total of 51 net counts), which allow us only to measure the normalization from which we derived the luminosities. By assuming the same power-law spectrum that describes $\mathrm{G} 1$, we measured a $L_{\mathrm{X}}^{0.5-2}=3 \times 10^{39} \mathrm{erg} / \mathrm{s}$.

Higdon (1996) evaluated intrinsic $B$ luminosities of G1 and G2: $L_{B}(\mathrm{G} 1)=6 \times 10^{9} L_{\odot}$ and $L_{B}(\mathrm{G} 2)=4.8 \times 10^{9} L_{\odot}$. There is no comparable measuremente in the literature for G3. Using the relation from Kim \& Fabbiano (2004) we expect that Low Mass X-ray Binaries $(\mathrm{LMXBs})$ contribute $L_{\mathrm{X}}^{\mathrm{G1}}(0.3-8 \mathrm{keV})=$ $5.4 \times 10^{39} \mathrm{erg} / \mathrm{s} ; L_{\mathrm{X}}^{\mathrm{G} 2}(0.3-8 \mathrm{keV})=4 . \times 10^{39} \mathrm{erg} / \mathrm{s}$ respectively. The observed $L_{X}$ is a factor of 7 and 4 higher in G1 and G2, respectively. At the XMM-Newton spatial resolution we cannot exclude the presence of a nuclear source in G1, however we know from the Chandra data that the emission is caused by a number of mostly off-center sources (see Fig. 7). Since the total G1 luminosity in the $2-10 \mathrm{keV}$ band has not varied between Chandra and XMM-Newton observations, we expect that the same sources contribute to the overall emission detected with XMM-Newton. Therefore, the relatively high $L_{X}$ in the companion galaxies, and in particular $\mathrm{G} 1$, is probably related to the interaction between the Sc galaxy G1 and the S0 companion G2, as also indicated by HI observations (Higdon 1996), which has resulted in morphological distortions, faint tidal tails, a higher SFR, and possibly a high number of HMXBs. Galaxy G3, now considered the "intruder" (Higdon 1996), has been poorly sampled at different wavelengths. Nevertheless, if it has the same corrected $\mathrm{L}_{B}$ as the other two companions, as appears to be the case according to their similar observed optical and IR magnitudes, its X-ray luminosity is in line with expectations from Kim \& Fabbiano (2004).

\section{Diffuse emission}

In spite of the relatively high number of net counts detected from the ring $(760 \pm 30)$, the interpretation of the results from spectral fitting is not straightforward. A simple power law fits the data, with a slope of $\Gamma=2.22[2.01-2.61]$, and a $\chi_{v}^{2}=0.72(24$ d.o.f.). While statistically acceptable, the fit has significant residuals, as already remarked in the analysis of Chandra data (Wolter \& Trinchieri 2004). We therefore used the same complex spectral fit used in Chandra, namely a powerlaw plus mekal model, to account for the excess below $1 \mathrm{keV}$. Since $n_{\mathrm{H}}$ and $k T$ are degenerate in the fit, we fixed the parameters to their Chandra values of $n_{\mathrm{H}}=2.3 \times 10^{21} \mathrm{~cm}^{-2}, \Gamma=2.3$, and $k T=0.2 \mathrm{keV}$, which are not far from those from a formal best-fit solution with fixed slope. Only the normalizations of the two models were allowed to be free parameters. The fit has a $\chi_{v}^{2}=0.75$ ( 25 d.o.f.). The resulting "gas" components has $L_{\mathrm{X}}^{0.5-2}=3 \times 10^{40} \mathrm{erg} / \mathrm{s}$, as in Chandra, while the "unresolved binary component" has $L_{\mathrm{X}}^{0.5-2}=4.3 \times 10^{40} \mathrm{erg} / \mathrm{s}$, and $L_{\mathrm{X}}^{2-10}=3.0 \times 10^{40} \mathrm{erg} / \mathrm{s}$, which is about a factor of 2 higher than in the Chandra spectrum. The higher power-law contribution relative to Chandra might partly be caused by the wings of the bright sources (especially N.10), which cannot be excluded as efficiently as in Chandra. The excess is indeed only $10-15 \%$ of the total $L_{\mathrm{X}}^{2-10}$ of the sources detected by XMM-Newton. The gas luminosity should be regarded as merely indicative of the true value, since the Chandra and XMM-Newton extraction regions used in its evaluation differ. For the Chandra data, we selected mainly the southwest portion of the ring and excluded a smaller fraction of the region considered (excluded regions have $\sim 1^{\prime \prime}$ radius). In XMM-Newton, the extraction region encompasses the whole ring, but the regions used to exclude sources are significantly larger and cover a large fraction of the brightest (southwest) region of the ring itself. With this caveat in mind, we evaluated the total gas luminosity in the ring by correcting the XMM-Newton result for the area lost due to sources, assuming the average surface brightness of the ring, which probably underestimates the true value, and derived $L_{\mathrm{X}}^{0.5-2}=3.5 \times 10^{40} \mathrm{erg} / \mathrm{s}$.

The spectral analysis of the "in" region (see Fig. 3) indicated that two components are needed: a plasma model, with a temperature of $k T=0.16[0.12-0.21] \mathrm{keV}$, similar to the ring value, and a power law to account for high energy residuals, which we parameterized with a slope fixed at $\Gamma=2\left(\chi_{v}^{2}=0.99\right.$ (26 d.o.f.)). A single mekal fit does not provide a good fit: it has a $\chi_{v}^{2}>5$; a single power law model also does not provide a good fit: it has $\chi_{v}^{2}=1.27$ (27 d.o.f.) and shows a bad distribution of the residuals. Formally, the luminosities in the two components are $L_{X}(0.5-2)=2.9 \times 10^{40} \mathrm{erg} / \mathrm{s}$ for the gas and $L_{X}(0.5-2 / 2-10)=$ $2.7 / 2.8 \times 10^{40} \mathrm{erg} / \mathrm{s}$ for the power law. This latter result is surprising since we do not expect such a significant contribution from binary sources: HMXBs ignited by the shock wave should no longer be active and there is no evidence of a young stellar population as there is in the ring, while LMXBs should not yet have formed after the burst. However, this component could instead correspond to a residual background: for instance, we expect a contribution from the PSF wings of the bright sources in the ring, which have been excluded only partially (the ring width only includes about $70 \%$ of the encircled energy for sources in the middle of the ring). We did not expect to find hot gas in the 
Table 5. The gas component parameters for the ring and the "in" region of the Cartwheel, computed from the summed EPIC-pn datasets, plus relative densities, volumes, and masses of gas.

\begin{tabular}{|c|c|c|c|c|c|c|c|c|}
\hline & \multicolumn{3}{|c|}{ Gas } & $n_{\mathrm{H}}$ & $\chi^{2}$ (d.o.f.) & $n$ & $V$ & $M$ \\
\hline & $k T$ & $\begin{array}{c}\text { pn Count rate } \\
(0.3-2 \mathrm{keV}) \\
\times 10^{-3}\end{array}$ & $\begin{array}{c}\text { Flux } \\
(0.5-2 \mathrm{keV}) \\
\times 10^{-14} \mathrm{erg} / \mathrm{cm}^{2} / \mathrm{s}\end{array}$ & $\times 10^{21} \mathrm{~cm}^{-2}$ & & {$\left[\mathrm{~cm}^{-3}\right]$} & {$\left[\mathrm{cm}^{3}\right]$} & {$\left[M_{\odot}\right]$} \\
\hline Ring & $0.20[0.16-0.22]$ & $10.09 \pm 0.43$ & 1.18 & 0.23 & $0.73(24)$ & $2.4 \times 10^{-3}$ & $2.03 \times 10^{68}$ & $4.7 \times 10^{8}$ \\
\hline "In" & $0.16[0.12-0.21]$ & $7.02 \pm 0.37$ & 1.03 & $2.9 \pm 0.9$ & $0.99(26)$ & $2.2 \times 10^{-3}$ & $1.50 \times 10^{68}$ & $2.8 \times 10^{8}$ \\
\hline
\end{tabular}

inner part of the Cartwheel. It would be tempting to associate the gas to the inner ring or a nucleus, since the inner ring was found to be bright at IR wavelengths (Charmandaris et al. 1999). However, our data are of insufficient spatial resolution to ascertain whether the hot gas emission is associated with a particular region of the galaxy.

In summary, the total luminosity of the Cartwheel gas computed using a mean temperature of $k T=0.2 \mathrm{keV}$, is $L_{\mathrm{X}} \sim$ $6.4 \times 10^{40} \mathrm{erg} \mathrm{s}^{-1}$, about $60 \%$ of which originates in the ring alone.

The mass of this gas is given in Table 5, separately for the ring and inner regions. We used the average gas density derived from the spectral parameters assuming that a fully ionized gas $\left(N_{\mathrm{e}}=N_{\mathrm{H}}\right)$ is distributed in a volume approximated by a toroid, for the ring, with $r_{\text {in }}=25^{\prime \prime}, r_{\text {out }}=40^{\prime \prime}$, and $r_{\text {torus }}=7.5^{\prime \prime}$, plus a cylinder, for the inner gas, with $r_{b}=25^{\prime \prime}$, and $h=15^{\prime \prime}$. Overall, the total mass in gas is $M=7.5 \times 10^{8} \eta^{1 / 2} M_{\odot}$ in the Cartwheel.

In a study of the ionized hot gas of a few young galaxies characterized by an intense star-formation activity, Tüllmann et al. (2006) calculated gas masses of about $10^{8} M_{\odot}$. Similar values have also been found, for instance, for the spiral galaxy M $83\left(M=2.2 \times 10^{8} M_{\odot}\right.$ for a filling factor of $\eta=1$, Ehle et al. 1998) or the interacting galaxy NGC 3395 in Arp 270 ( $M=1.02 \times 10^{8} M_{\odot}$ for $\eta=1$, Brassington et al. 2005). In this respect, the Cartwheel galaxy appears to have a higher gas mass than these other young/interacting galaxies.

We estimate also that the thermal energy associated with the hot plasma for $\eta=1$ is $E_{\mathrm{X}}=3 n_{\mathrm{e}} \eta V k T=4.6 / 3.2 \times 10^{56}$ erg for the ring/inner region. This again appears to be high, since it is only a factor of 2 lower than the thermal energy $E_{\mathrm{X}}=1.5 \times 10^{57}$ erg estimated for the starburst galaxy NGC 3256 (Lira et al. 2002, a spectacular merger, one of the most IR luminous system in the local universe). While caution should be taken when interpreting our results, which depend on several assumptions about the spectral parameters and corrections applied (see previous discussion), the high values derived could be due to the significant clumpiness of the medium.

Finally, we extract the spectrum of gas in the group within a circular region of $60^{\prime \prime}$ radius (see Fig. 3 ), excluding the contributions of the galaxies and the background sources. Given the presence of bright sources in the area, we must again take into account their contribution outside the excluded regions. In particular, for the bright source XMM11 to the NE, we used an exclusion region of radius $8^{\prime \prime}$. A larger radius would reduce too much the area available to measure the group emission. The contamination from XMM11 is therefore on the order of $25 \%$ of its flux. We extracted its spectrum and derived a powerlaw index $\Gamma=2.0$, which we added to the model spectrum for the group emission, described by a mekal model, to account for the high energy part of the spectrum. We fixed the normalization of the power law to be $25 \%$ of the flux of XMM11.

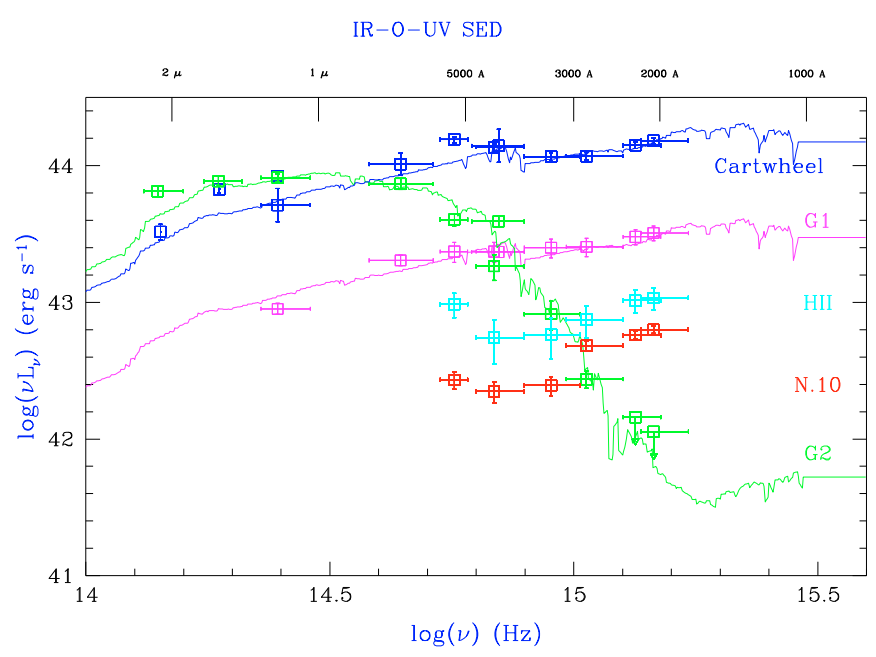

Fig. 8. SED of Cartwheel ring, G1, G2, and HII and N.10, see text for description. Data from OM, Marcum et al. (1992), 2MASS.

The resulting group gas has a temperature $k T=0.21[0.15-$ $0.27] \mathrm{keV}$ and a luminosity $L_{\mathrm{X}}^{0.5-2}=9 \times 10^{39} \mathrm{erg} \mathrm{s}^{-1}$. These values are much lower than typical values of compact groups $(k T \sim$ $1 \mathrm{keV}$ and $10^{42} \mathrm{erg} \mathrm{s}^{-1}$, Ponman et al. 1996; Mulchaey et al. 2000). However, observations of dynamically young, spiraldominated systems indicate significantly softer and lower $L_{X}$ IGM (SCG0018-4854: Trinchieri et al. 2008; HCG16: Belsole et al. 2003; HCG 80: Ota et al. 2004).

The measurements of the gas in the Cartwheel group is formally consistent with the extrapolation to low temperatures of the $L_{X}-k T$ relation for groups and clusters (Mulchaey et al. 2000). Nevertheless, given the large uncertainties and scatter in the extrapolation, this result is not conclusive.

\section{IR to UV SED}

Using data from the OM instrument in the six UV/optical bands observed, we studied the broad-band energy distribution of the ring, of galaxies G1 and G2, and of two regions in the ring, corresponding to the brightest ULX, N.10, and to the brightest IR hotspot (labelled HII). The fluxes obtained in each filter are listed in Table 6. We added the IR fluxes from the literature, in particular the 2MASS data (total magnitude, Skrutskie et al. 2006) for the Cartwheel and G2. We emphasize that the 2MASS measurement includes the entire Cartwheel, so they are not strictly comparable to the values reported in Table 6 , which refer to the ring only. We also included the ring, G1, and G2 values in $B$ and $K$ band from Marcum et al.(1992).

Figure 8 shows the Spectral Energy Distributions for the different sources considered, from Table 6 data. We also show two 
Table 6. Optical and UV flux of ULXs sources and HII region of the southern ring of the Cartwheel, G1, and the total ring.

\begin{tabular}{c|cc|cc|cc|cc|cc|cc}
\hline \hline & \multicolumn{2}{|c|}{$V$} & \multicolumn{2}{|c|}{$B$} & \multicolumn{2}{c|}{$U$} & \multicolumn{2}{c|}{$U V W 1$} & \multicolumn{2}{c}{$U V M 2$} & \multicolumn{2}{c}{$U V W 2$} \\
& Flux & Mag & Flux & Mag & Flux & Mag & Flux & Mag & Flux & Mag & Flux & Mag \\
& $\times 10^{-12}$ & & $\times 10^{-12}$ & & $\times 10^{-12}$ & & $\times 10^{-12}$ & & $\times 10^{-12}$ & & $\times 10^{-12}$ \\
\hline $\boldsymbol{N 7 + N 9}$ & 0.93 & 18.37 & 0.69 & 19.07 & 0.77 & 18.09 & 1.26 & 17.31 & 1.57 & 17.03 & 1.82 & 16.94 \\
N10 & 0.69 & 18.68 & 0.57 & 19.27 & 0.63 & 18.32 & 1.23 & 17.34 & 1.47 & 17.12 & 1.61 & 17.05 \\
N13+N14 & 0.96 & 18.34 & 0.78 & 18.94 & 0.92 & 17.91 & 1.66 & 17.01 & 2.03 & 16.77 & 2.30 & 16.67 \\
N16+N17 & 0.55 & 18.93 & 0.48 & 19.47 & 0.49 & 18.59 & 1.09 & 17.45 & 1.37 & 17.17 & 1.53 & 17.08 \\
HII region & 2.47 & 17.31 & 1.41 & 18.03 & 1.48 & 17.39 & 1.91 & 16.67 & 2.63 & 16.48 & 2.75 & 16.48 \\
G1 & 6.02 & 16.32 & 6.08 & 16.72 & 6.43 & 15.80 & 6.55 & 15.52 & 7.71 & 15.32 & 8.20 & 15.29 \\
G2 & 10.3 & 15.76 & 4.73 & 16.99 & 2.14 & 17.00 & 0.66 & 18.01 & 0.13 & 19.78 & $<0.08$ & $>20.00$ \\
Ring & 39.7 & 14.29 & 34.9 & 14.82 & 29.8 & 14.13 & 30.1 & 13.86 & 36.0 & 13.65 & 39.0 & 13.60 \\
\hline
\end{tabular}

models: 1) a model spectrum typical of an old elliptical (generated with the GRASIL code; Silva et al. $1998^{4}$ ), normalized to the data of G2; this model describes the shape of this galaxy spectrum well; 2) the spectrum resulting from a burst of star formation (courtesy of Paolo Franzetti, private communication), constructed with the PEGASE code (Rocca-Volmerange \& Fioc 1999) with a star-formation history from Gavazzi et al. (2002). This model describes the Cartwheel ring and, at a slightly lower total luminosity, G1. The age of the burst is $400 \mathrm{Myr}$, which is comparable to the age of the collision (300 Myr from Fosbury \& Hawarden 1977; Marcum et al. 1992; and Higdon 1996), while the mean stellar age is about $\sim 40$ Myr. The "effective" optical thickness (related to the extinction as in Guiderdoni \& RoccaVolmerange 1987) and the metallicity are computed in an autoconsistent way by the model itself. The stellar mass of the model is $M=4.9 \times 10^{9} M_{\odot}$, which implies that the stars produced in the ring by the shock represent a small fraction of the total mass of the entire galaxy before the encounter, estimated to be $M=5 \times 10^{11} M_{\odot}($ Fosbury \& Hawarden 1977). The normalization of the model provides a measure of the star formation rate (SFR) corresponding to $S F R=23 M_{\odot} / \mathrm{yr}$ for the Cartwheel and $S F R=5 M_{\odot} / \mathrm{yr}$ for G1. The estimate agrees well with other measures of the SFR in the Cartwheel (Mayya et al. 2005; Wolter \& Trinchieri 2004). We note that different combinations of burst age and SFR could reproduce the observed spectrum. However, the mean stellar age is consistently on the order of a few $10^{7} \mathrm{yr}$. The model also appears to provide an accurate description of G1, although it might not be entirely appropriate to the evolutionary state of G1, since it should not have been involved in the collision.

The HII region and N.10 are similar in shape to the total Cartwheel ring, if anything even bluer. However, given the physical size of the extraction regions $(\geq 1 \mathrm{kpc})$ we expect a large contamination from the ring in which they are embedded, and it is not straightforward to evaluate independently their colors.

\section{Summary}

We have observed the Cartwheel with XMM-Newton at two different epochs separated by 5 months. We detected 7 sources in the ring, plus one in the inner part, which however could be a background source (N.11), all at the luminosity level of ULXs. The relatively large region $(\sim 6 \mathrm{kpc})$ in which these luminosity measurements were obtained could include a large number of fainter sources, although from the comparison with Chandra data

\footnotetext{
4 See also

http: //cass.ucsd.edu/SWIRE/mcp/templates/

swire_templates.html
}

we know that there are only one or two dominant individual objects in each detection. This is also supported by the observed variability of all ULXs: at least 4 of them have varied in the 4 years between the Chandra and the first XMM-Newton observation, and at least 3 in only 5 months, in addition to the source N.10 discussed in Wolter et al. (2006).

The variability amplitude and frequency suggest an association with X-ray binaries. The optical SED of the Cartwheel ring has very blue colors, consistent with a recent burst of SF activity, of age similar to previous estimates for the tidal encounter that generated the shock, producing stars of mean age of a few $10^{7} \mathrm{yr}$. Binaries are therefore likely to be HMXBs, since the presence of a sizable population of IMBHs is unlikely (King 2004).

Overall, the X-ray characteristics appear to be consistent with various formation and evolutionary models discussed for ring galaxies and particular the Cartwheel itself (e.g. Mapelli et al. 2008; King 2004; Marcum et al. 1992; Higdon 1996) where a radial shock, generated at the impact with the companion galaxy, is driven into a predominantly gaseous disk in which star formation is triggered. The shock itself, propagating at a speed equal to the expansion velocity of the ring $\left(\sim 60 \mathrm{~km} \mathrm{~s}^{-1}\right.$, from HI data; Higdon 1996, or even lower from $\mathrm{H} \alpha$ measurements; Amram et al. 1998) would heat the gas to a temperature of $k T \leq 0.05 \mathrm{keV}$, lower than measured. However, the shock should be responsible for the enhanced star formation, which in turn heats the gas (Wolter et al. 1999).

The gas of the Cartwheel has a very low temperature and appears to be quite luminous $\left(L_{X}=6 \times 10^{40} \mathrm{erg} / \mathrm{s}\right)$. We derive a relatively large gas mass, both in the ring and in the inner disk region.

We also detect a hot intergroup medium of low temperature. Although other systems exist with similar low temperatures and luminosities, the statistical significance of the detection does not allow us to complete a meaningful comparison with them.

Acknowledgements. It is always a pleasure to acknowledge useful discussions with Angela Iovino. The analysis of OM data has greatly benefited form the help of Marcella Longhetti, Mari Polletta, Paolo Franzetti, Ruth Grützbach, and Marco Scodeggio. We thank an anonymous referee for very useful comments that helped us to improve the paper significantly. We acknowledge partial financial support from the Italian Space Agency under contract ASI-INAF I/023/05/0. This work is based on observations obtained with XMM-Newton, an ESA science mission with instruments and contributions directly funded by ESA Members States and the USA (NASA). This research has made use of SAOImage DS9, developed by Smithsonian Astrophysical Observatory.

\section{References}

Amram, P., Mendes de Oliveira, C., Boulesteix, J., \& Balkowski C. 1988, A\&A, 330,881

Appleton, P. N., \& Struck-Marcell, C. 1987, ApJ, 318, 103 
Belsole, E., Sauvageot, J.-L., Ponman, T. J., \& Bourdin, H. 2003, A\&A, 398, Brassington, N., Read, A. M., \& Ponman, T. J. 2005, MNRAS, 360, 810 Charmandaris, V., Laurent, O., Mirabel, I. F., et al. 1999, A\&A, 341, 69 Dickey, J. M., \& Lockman, F. J. 1990, ARA\&A, 28, 215

Ehle, M., Pietsch, W., Beck, R., \& Klein, U. 1998, A\&A, 329, 39

Fosbury, R. A., \& Hawarden, T. G. 1977, MNRAS, 178, 473

Gao, Y., Wang, Q. D., Appleton, P. N., \& Lucas, R. A. 2003, ApJ, 596, 171 Gavazzi, G., Bonfanti, C., Sanvito, G., Boselli, A., \& Scodeggio, M. 2002, ApJ, 576,135

Gil de Paz, A., Boissier, S., Madore, B. F., et al. 2007, ApJS, 173, 185

Guiderdoni, B., \& Rocca-Volmerange, B. 1987, A\&A, 186, 1

Higdon, J. L. 1996, ApJ, 467, 241

Iovino, A. 2002, AJ, 124, 2471

Kim, D.-W., \& Fabbiano, G. 2004, ApJ, 611, 846

King, A. R. 2004, MNRAS, 347, L18

Lira, P., Ward, M., Zezas, A., Alons-Herrero, A., \& Ueno, S. 2002, MNRAS, 330,259

Marcum, P. M., Appleton, P. N., \& Higdon, J. 1992, ApJ, 399, 57

Mapelli, M., Moore, B., Giordano, L., et al. 2008, MNRAS, 383, 230
Mayya, Y. D., Bizyaev, D., Romano, R., Garcia-Barreto, J. A., \& Vorobyov, E. I. 2005, ApJ, 620, 35

Mulchaey, J. S. 2000, ARA\&A, 38, 289

Ota, N., Morita, U., Kitayama, T., \& Oashi, T. 2004, PASJ, 56, 753

Ponman, T. J., Bourner, D. J., Ebeling, H., \& Böringer, H. 1996, MNRAS, 283, 690

Rocca-Volmerange, B., \& Fioc, M. 1999, Ap\&SS, 269, 233

Silva, L., Granato, G. L., Bressan, A., \& Danese, L. 1998, ApJ, 509, 103

Skrutskie, M. F., Cutri, R. M., Stiening, R., et al. 2006, AJ, 131, 1163

Struck, C., Appleton, P. N., Borne, K. D., \& Lucas, R. A. 1996, AJ, 112, 1868

Taylor, K., \& Atherton, P. D. 1984, MNRAS, 208, 650

Theys, J. C., \& Spiegel, E. A. 1976, ApJ, 208, 650

Trinchieri, G., Iovino, A., Pompei, E., et al. 2008, A\&A, 484, 195

Tüllmann, R., Pietsch, W., Rossa, J., Breitschwerdt, D., \& Dettmar, R. J. 2006, A\&A, 448, 43

Wolter, A., \& Trinchieri, G. 2004, A\&A, 426, 787

Wolter, A., Trinchieri, G., \& Iovino, A. 1999, A\&A, 342, 41

Wolter, A., Trinchieri, G., \& Colpi, M. 2006, MNRAS, 373, 1627

Zezas, A., Fabbiano, G., Baldi, A., et al. 2006, ApJS, 166, 211 